intervention and the labelling of a large number of normal infants with a condition they do not have.

Before embarking on such a course the baby should at the very least be observed, handled, and fed by experienced staff in a calm atmosphere, perhaps in a day care unit. This often resolves the problem and relieves the parents of much unnecessary anxiety. If this fails to resolve symptoms I concede that a trial with soy might be worthwhile in those few babies whose symptoms remain, but there is no convincing objective evidence that this is beneficial. Perhaps someone should try to find out.

\section{Screening for cystic fibrosis}

Sir,

In his commentary on our recent review paper $^{1}$ in which we advocate a programme of neonatal screening for cystic fibrosis (CF), Professor Peter Phelan rightly points out that data in the published reports are inconclusive. This is partly because of the difficulty in obtaining data which compare like populations. Professor Phelan himself compares the survival figures for 30 years ago (fewer than $10 \%$ alive at 5 years) when definitive diagnosis was often made at necropsy only, with major contemporary US and Australian statistics showing $80 \%$ survival to late adolescence. The Gibson-Cooke standardised sweat test was not available before 1959, and its widespread application has resulted in identification of milder cases and better understanding of the considerable variations in the natural history of CF which are independent of particular treatment regimens.

Our support for a neonatal screening programme is based as much on the value of a pre-symptomatic diagnosis to the individual parents and child as on the scanty useful incidence and mortality data in the reports. ${ }^{2}$ The fact that the majority of newly diagnosed children with CF are born into families without a previously affected child is of no help to those parents whose eldest CF child is diagnosed only after the birth of the second. We are less sanguine about the long term outlook for CF patients than Professor Phelan and conscious of the physical and emotional cost at which the improving prognosis is achieved. New approaches to treatment are needed, perhaps with particular attention to early nutritional support. A recent paper from another Australian centre indicates how, even in patients with advanced CF, substantial benefit can be achieved by an aggressive nutritional policy. ${ }^{3}$ We have not, contrary to the fear expressed by Professor Phelan, encountered parental rejection on the grounds of the diagnosis of CF. Our experience is that parents told (we hope sensitively, optimistically, but realistically) that their new born infant has CF nearly always form a very close bond with their baby: nor is rejection an observed phenomenon in the $15 \%$ or so who present with meconium ileus and are effectively self selected for neonatal screening.

We agree that measures of the progress of lung disease in early life are insensitive, and feel that Professor Phelan can not really be sure that his 39 patients with a family history, who were therefore detected early, were as- certained 'before the development of lung disease'. We also agree that the benefits of early diagnosis in a long term prospective study of survival may be small, but this is not a valid argument against such a study since only an unselected series can unequivocally show differences between centres and treatment regimens-the methods of selection for treatment at a particular centre being otherwise questionable.

The existence of a screening programme implies the existence of a regional centre for diagnosis with which a treatment centre is likely to be associated. Although Professor Phelan claims that $95 \%$ of all patients in Victoria are treated at his own (justly renowned) centre, simple population statistics suggest that this may not be so. In our own centre, which treats all patients from South Glamorgan (population approximately 400000) and an equal number from other health authorities, about 55 are resident in the area. This gives a prevalence of 1:7500. The population of Victoria is about 4000000 and the clinic in Melbourne has 336 patients, ie a prevalence of $1: 12000$ in a comparable ethnic group. Either the Cardiff survival figures are much better than those of Victoria (which we doubt) or about $30 \%$ of the Australian patients are being cared for, or dying undiagnosed, without reference to the regional centre. Perhaps there is therefore a particularly strong case for neonatal screening in Victoria, the results of which might well surprise us all.

\section{References}

1 Phelan P D. Commentary. Arch Dis Child 1982; 57: 779-80.

2 Dodge J A, Ryley H C. Screening for cystic fibrosis. Arch Dis Child 1982; 57: 774-80.

3 Shepherd R, Cooksley W G E, Cooke W D D. Improved growth and clinical, nutritional, and respiratory changes in response to nutritional therapy in cystic fibrosis. J Pediatr 1980; 97 : 351-7.

J A DODGE AND H C RYLEY

Departments of Child Health and Medical Microbiology, University of Wales, Heath Park, Cardiff CF4 $4 X N$

\section{Professor Phelan comments:}

In their letter Dodge and Ryley raise a number of important issues that warrant further comment. I am pleased that they agree that great difficulties are likely to be experienced in showing an improved outlook as a result of newborn screening for cystic fibrosis and they are almost certainly correct in predicting that demonstrable long term benefits of early diagnosis will, at the best, be small.

They suggest that parents appreciate early presymptomatic diagnosis. However, this remains to be proved by a properly conducted study. It is not reasonable to compare ill babies with meconium ileus with those diagnosed pre-symptomatically. It seems crucial to show, before newborn screening is widely introduced, that pre-symptomatic diagnosis is beneficial to parents and child and that it does not disturb developing family 